\title{
Research and Design of Classroom Integrated Management System Based on Internet of Things
}

\author{
Zhongwei Zhao ${ }^{1, \mathrm{a}}$, He Gong ${ }^{1, \mathrm{~b}^{*}}$, Yizhe Sun ${ }^{1, \mathrm{c}}$ and Bin Liu ${ }^{1, \mathrm{~d}}$ \\ ${ }^{1}$ Jilin Agricultural University, Changchun 130118, Jilin, China \\ a1411864225@qq.com, bonghe@jlau.edu.cn, ‘631975748@qq.com, diubin062690@163.com \\ * The corresponding author
}

Keywords: Internet of Things; ZigBee; Classroom Management; Ringing Bells

\begin{abstract}
Considering the problems of flexible control of classroom bell time, the idle state information of the classroom is not easy to obtain and difficult to detect the classroom environment information, a classroom integrated management system based on internet of things is researched and designed. By using the ZigBee collection node to detect the classroom use of environmental information, the ZigBee network collects information sent to the server, and the intelligent management system analyzes these data to supervise of the classroom. According to the test, the system is a good solution to the traditional classroom management in the face of the ringing time fixed uniform, improving the level of automation of classroom management.
\end{abstract}

\section{Introduction}

There are shortcomings in the classroom management of traditional colleges and universities, such as the lack of resources, such as each classroom is based on a fixed period of time to set the bell, this setting is easy to interfere with the self-study students, and the free classroom Ringing settings also wasted resources. Students must find their own free classroom, which greatly wasted the student's time. At the same time, because the real-time monitoring of classroom environmental information, resulting in a low level of fire safety.

With the increasing demand for intelligent classroom management in colleges and universities, more and more research scholars have applied the Internet of Things technology and classroom management in colleges and universities. Such as the literature [1] through the use of computers to control the ring bell, you can always choose the bell time. The literature [2-3] designed a radio bell control system that automatically changed the ringing time with the seasonal changes. The literature [4] designed a precise control of the use of GPS time, easy debugging smart bell control system. The literature [5] through the Internet of things integration of classroom information, reducing the installation of the installation and commissioning system costs. The literature [6] through the Internet of Things technology designed specifically for the management and control of a variety of intelligent perception mechanism of work, by modifying the intelligent perception mode for the staff of the space to provide intelligent service classroom management system.

This paper presents a new classroom management system based on the Internet of Things. The system solves some shortcomings of the traditional classroom management system, and makes the teaching environment more humane. The application of the Internet of things in the classroom and classroom management, monitoring real-time environmental information and usage, and according to the actual needs of rational management of classroom bell equipment, improve security level, fire, combined with large data analysis to facilitate students to quickly find the spare room.

\section{Overall System Framework}

The system consists of terminal node, ZigBee coordinator and server. Terminal nodes are deployed in various classrooms, responsible for ringing, news notification, fire safety monitoring and other functions. The main role of the ZigBee coordinator is to establish a wireless network and transfer data between the server and the terminal nodes. The server controls the operation of the nodes of the 
wireless network through the application running on it and also manages the data of the entire system. Functions include the timetable, classroom use status, indoor environment and another information query, change, storage. The overall structure of the system is shown in Fig. 1.

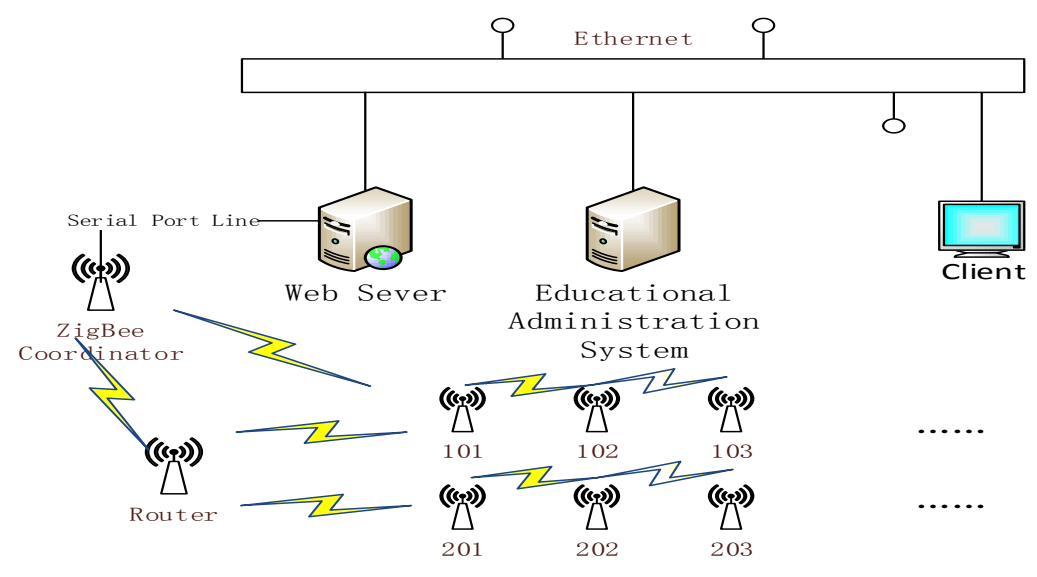

Figure 1. Overall structure of the system

\section{Hard ware Design}

Terminal Control Node Design. Terminal control node consists of main control chip, sensor, real-time clock, external memory chip, extended interface, voice module, terminal control node circuit as shown in Fig. 2. The smoke sensor outputs the digital signal to the master chip JN5148. The master chip judges whether the smoke in the classroom reaches the alarm threshold by analyzing the level of the level. If the alarm threshold is reached, the signal is sent to the voice module to send out the alarm signal. The pyroelectric infrared sensor is a sensor that detects the infrared rays emitted by a person or an animal and outputs an electrical signal to determine whether someone is using the classroom and upload the data to the server via the master chip for processing.

The voice module consist of voice synthesis module, signal selection chip, MP3 module and active speaker. Signal selection chip by analyzing the master chip JN5148 sent over the information, you can choose to use the voice synthesis module for broadcast or use MP3 module for broadcast. When you can rest during the class, you can use the MP3 module to play the soothing music to make the students relax between classes. Normal upper and lower classes will choose to use the voice synthesis module for broadcast. The hardware of the terminal control node is shown in Fig. 3.

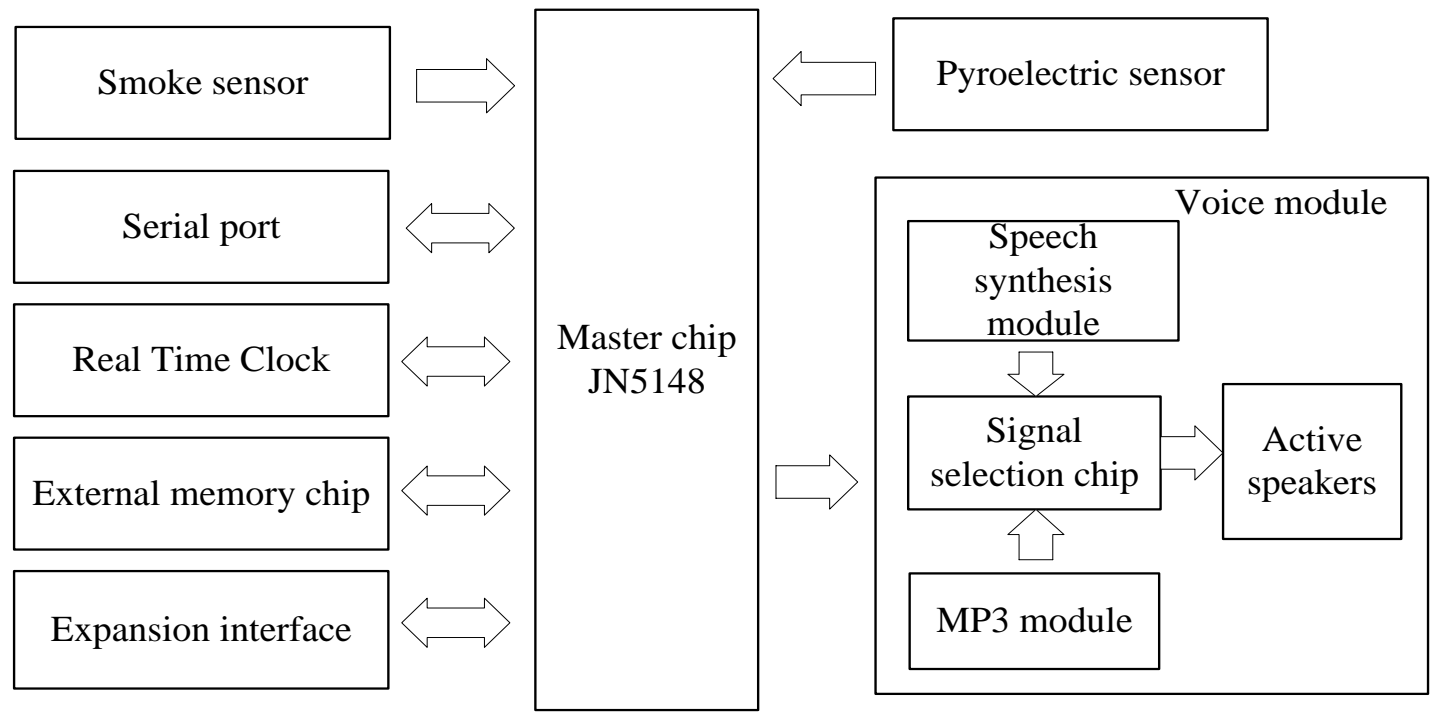

Figure 2. Terminal control node circuit 


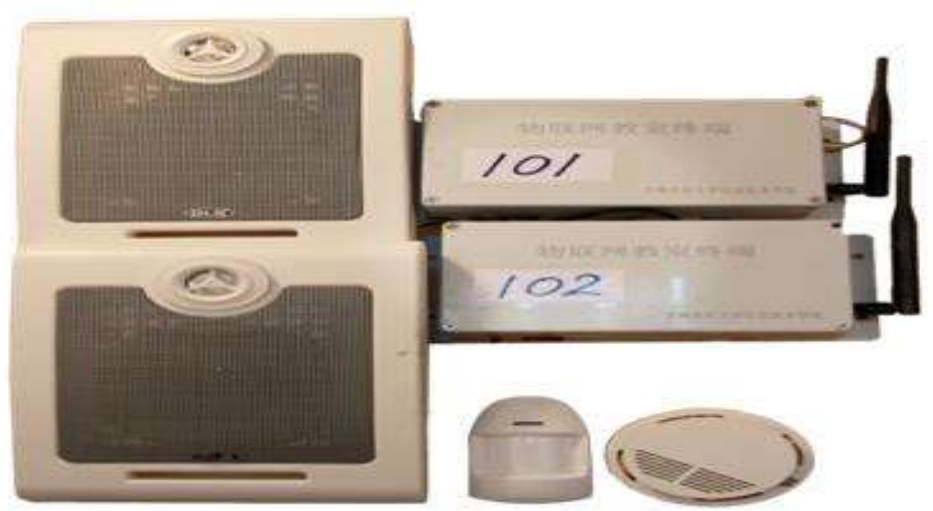

Figure 3. The hardware of the terminal control node

ZigBee Coordinator. The coordinator is the core of the entire ZigBee network and is responsible for the task of establishing and managing the network. In this system, the coordinator can be connected to the server through the serial port to realize the communication between the server and the ZigBee network. The coordinator sends the command or data sent by the server application to the corresponding classroom node or receives the data sent from the classroom node and then uploads to the server, sometimes instead of the server to perform some of the underlying operations, with only a few instructions, to complete their own.

\section{Software Design}

PC Software design. The PC software design uses the B/S architecture, namely the browser and the server unifies the method to design. The core of the system will be concentrated on the server, users only need to be able to run the browser device can view the classroom management information, that is, users can view information, but also reduce maintenance costs. At the same time the host computer data connected to the entire campus network, according to the data on the table analysis, intelligent choice of classrooms for voice broadcast, while the analysis of classroom use, to facilitate learning or management personnel to view. The PC function design is shown in Fig. 4.

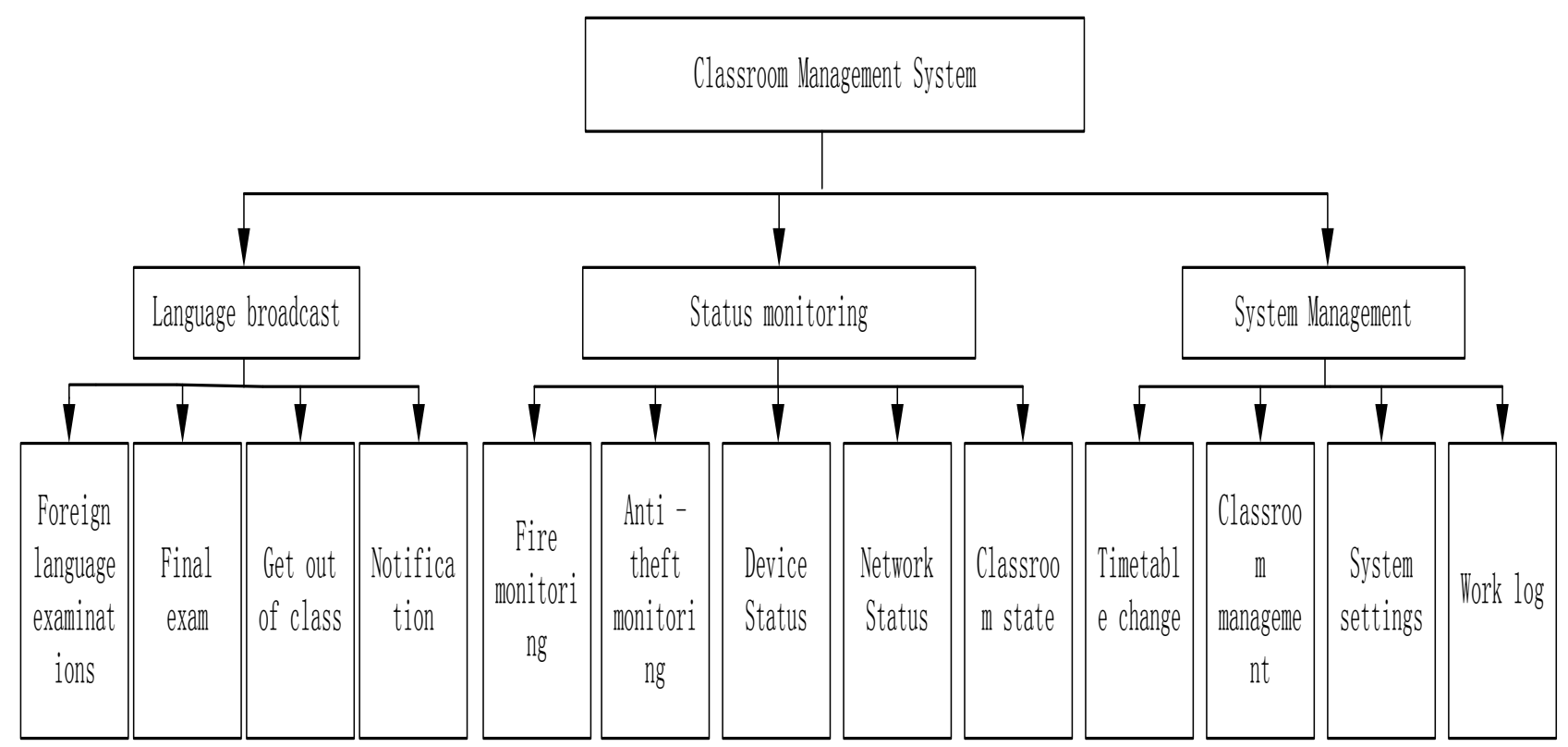

Figure 4. The PC function

Administrators login through the browser to view and manage the classroom information, students can query through the entrance of the teaching building or landing campus network query 
free classrooms. The user login interface is shown in Fig. 5.
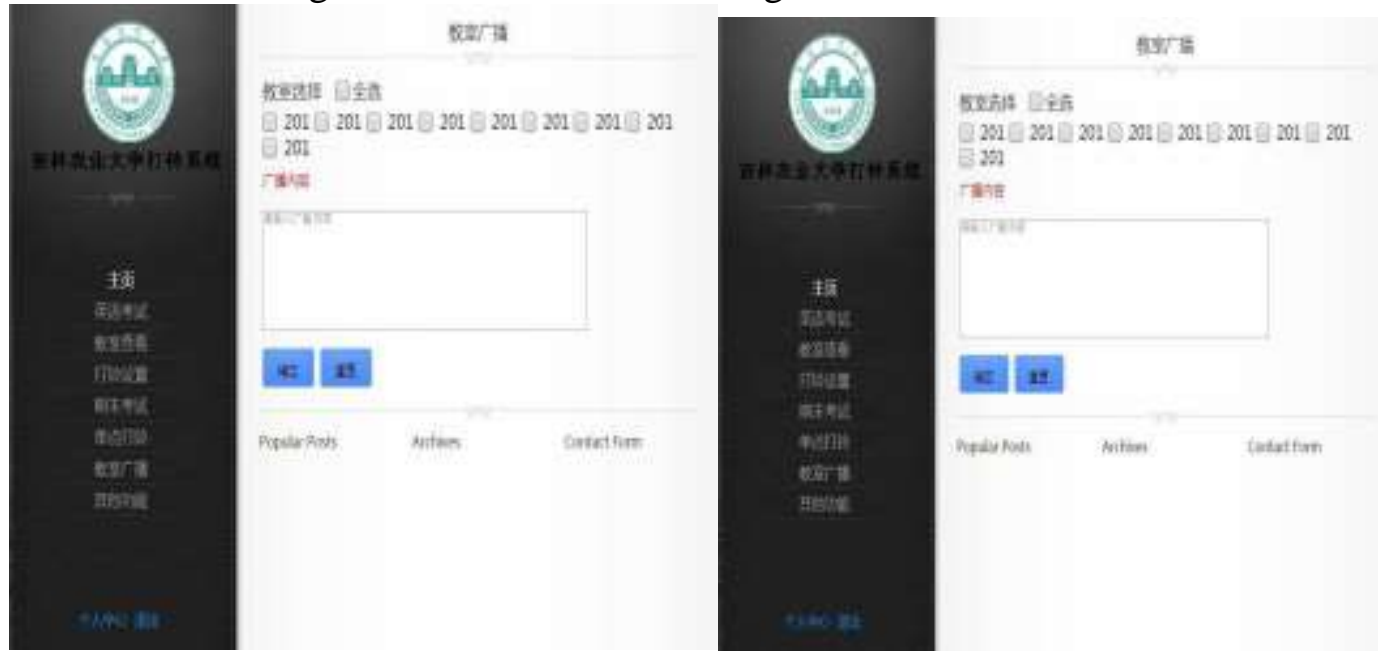

Figure 5. The user login interface

Terminal Node Software Design. The terminal controls the time it takes for the node to periodically read the clock module, and performs the specific task in conjunction with the contents of the ringing task table. Task table by the task time, task type, task content, etc., when the clock time and task table of the same time, read the task type and specific content execution, such as the need to play voice prompts, then open the speaker, send to the voice synthesis module needs Play the voice data and wait for the playback to turn off the speaker. The terminal control node design process is shown in Fig. 6.

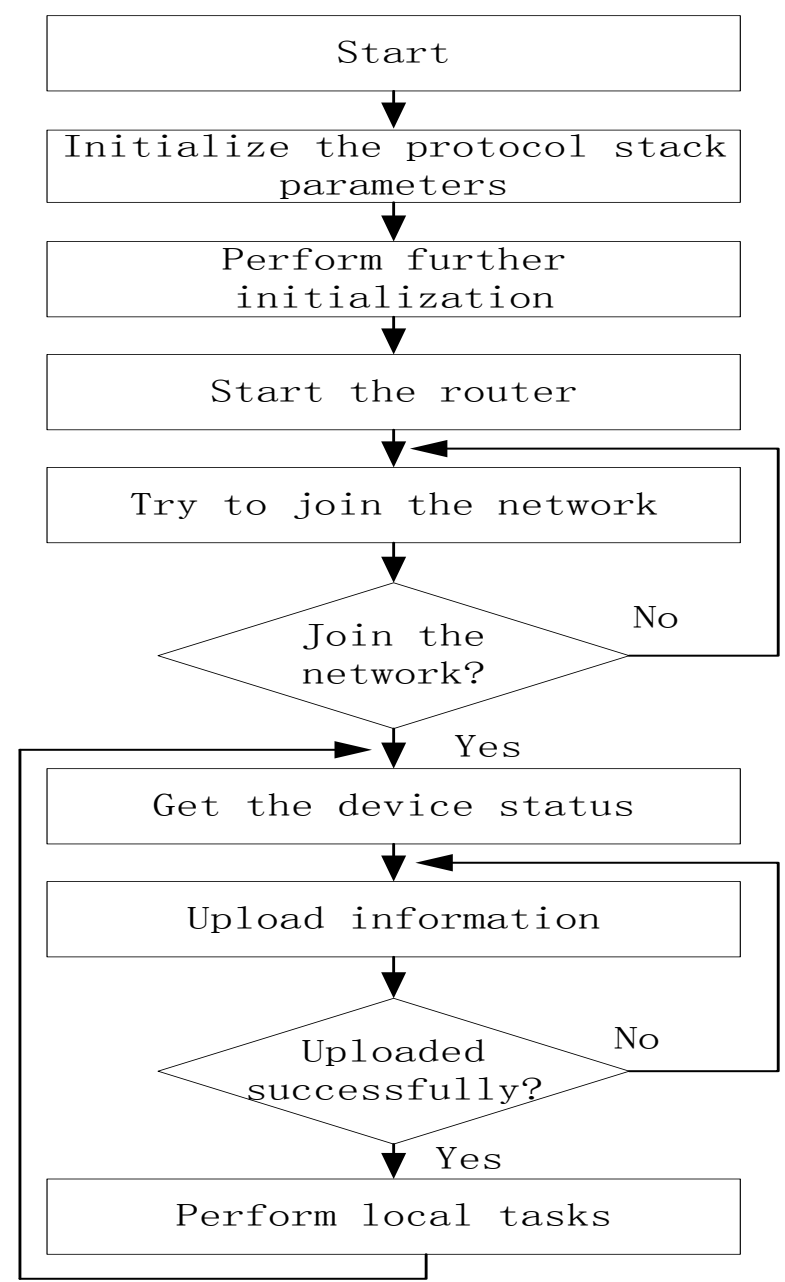

Figure 6. The terminal control node design process 
Task schedule is divided into class schedule, test schedule, custom schedule. There are two sets of templates for the summer schedule and the winter schedule. The examination schedule has the final exam and the 46 test table. The custom schedule can change the default settings of these tables by instruction to realize the custom function.

After a day's task, the system automatically updates the next day's task list according to the educational administration timetable information and task table template.

When you need to send a temporary notification, you can always send the text or send control commands over the network to play the audio files on the memory card. When the network is not good, the classroom nodes can be set according to the pre-automatic operation, network recovery and timely update the latest version of the task table.

\section{Summary}

This paper studies the design and use of the classroom integrated management system based on the Internet of Things, which effectively solves the problems such as the uniformity of the playing time in the traditional classroom management, the lack of flexibility, the inability to recognize the use of the classroom and the improvement of classroom management Intelligent level.

\section{Acknowledgments}

The authors wish to express their gratitude to the projects: Research and Design of Classroom Integrated Management System Based on Internet of Things from Construction and Application of Internet + Deer Industry Information Platform(No. 20170204038NY). At the same time, the other authors also thank the corresponding author, He Gong, for his assistance in submitting this paper.

\section{References}

[1] H. Sun, Research on Remote Synchronization Control Bell System, J. Science and Technology Consulting Herald.2007(26): 14-15. (In Chinese)

[2] T.S. Jiang, Wireless Control System for Electric Bell Based on STC12C5410AD, J. Journal of Hunan Environment-Biological Polytechnic. 2008(3): 20-23. (In Chinese)

[3] M.C. Zong, F. Wen, Design of Automatic Bell Controller, J. Mechanical Engineering \& Automation.2013(2): 153-155. (In Chinese)

[4] Y.W. Xu, Y. Li, Design of Multifunctional Electrical Bell Based on the Single Chip Microcomputer Wireless Control, J. Electronic Test. 2013(9): 21-24. (In Chinese)

[5] X. Zhang, Z.D. Hua and Y. Yang, CC2530 based Inteligent Control System of The Classroom Building, J. Industrial Design. 2017,(07):183-184. (In Chinese)

[6] J.J. Zheng, K.L. Zheng, Research and Design of Smart Campus Architecture Based on Internet of Things Technology, J. Journal of Tongren University.2016,18(04):67-73. (In Chinese)

[7] H.L. Hou, X.X. Zhang and F.M. Wang, Summary of ZigBee Wireless Transmission Technology, J. Shanxi Electronic Technology.2011(4): 84-86. (In Chinese)

[8] J.B. Bai, X.S. Zhang and S.K. Lu, Technology and Its Application in Building Automation System, J. Intelligent Building \& Smart City.2006(1): 102-104. (In Chinese) 